\title{
The genome of black cottonwood, Populus trichocarpa (Torr. \& Gray)
}

G. A. Tuskan,,$^{1,3}$ S. DiFazio, ${ }^{1,4^{*}}$ S. Jansson, ${ }^{9^{*}}$ J. Bohlmann,$^{5^{*}}$ I. Grigoriev,$^{8^{*}}$ U. Hellsten,,$^{8^{*}}$

6 N. Putnam, ${ }^{8^{*}}$ S. Ralph, ${ }^{5^{*}}$ S. Rombauts, ${ }^{10^{*}}$ A. Salamov, ${ }^{8^{*}}$ J. Schein, ${ }^{11^{*}}$ L. Sterck, ${ }^{10^{*}}$ A. Aerts, ${ }^{8}$ R. R. Bhalerao, ${ }^{9}$ R. P. Bhalerao, ${ }^{12}$ D. Blaudez, ${ }^{13}$ W. Boerjan, ${ }^{10}$ A. Brun, ${ }^{13}$ A. Brunner, ${ }^{14}$ V. Busov, ${ }^{15}$ M. Campbell, ${ }^{16}$ J. Carlson, ${ }^{17}$ M. Chalot, ${ }^{13}$ J. Chapman, ${ }^{8}$ G.-L.

9 Chen, ${ }^{2}$ D. Cooper, ${ }^{5}$ P.M. Coutinho, ${ }^{19}$ J. Couturier, ${ }^{13}$ S. Covert, ${ }^{20}$ Q. Cronk, ${ }^{6}$ R. Cunningham, ${ }^{1} \mathrm{~J}$. Davis ${ }^{22} \mathrm{~S}$. Degroeve,${ }^{10}$ A. Déjardin, ${ }^{23} \mathrm{C}$. dePamphilis, ${ }^{18} \mathrm{~J}$. Detter, ${ }^{8} \mathrm{~B}$. Dirks, ${ }^{24}$ I. Dubchak, ${ }^{8,25}$ S. Duplessis, ${ }^{13}$ J. Ehlting, ${ }^{6}$ B. Ellis, ${ }^{5}$ K. Gendler,${ }^{26}$ D. Goodstein, ${ }^{8}$

12 M. Gribskov, ${ }^{27}$ J. Grimwood, ${ }^{28}$ A. Groover, ${ }^{29}$ L. Gunter, ${ }^{1}$ B. Hamberger, ${ }^{6}$ B. Heinze ${ }^{30}$ Y. Helariutta, ${ }^{31,12,33}$ B. Henrissat, ${ }^{19}$ D. Holligan, ${ }^{21}$ R. Holt,,${ }^{11}$ W. Huang, ${ }^{8}$ N. Islam-Faridi, ${ }^{34} \mathrm{~S}$. Jones,${ }^{11} \mathrm{M}$. Jones-Rhoades ${ }^{35} \mathrm{R}$. Jorgensen ${ }^{26} \mathrm{C}$. Joshi, ${ }^{15} \mathrm{~J}$. Kangasjärvi, ${ }^{32} \mathrm{~J}$. Karlsson, ${ }^{9}$

15 C. Kelleher, ${ }^{5}$ R. Kirkpatrick, ${ }^{11}$ M. Kirst ${ }^{22}$ A. Kohler, ${ }^{13}$ U. Kalluri, ${ }^{1}$ F. Larimer, ${ }^{2}$ J. LeebensMack, ${ }^{21}$ J.-C. Leplé, ${ }^{23}$ P. Locascio, ${ }^{2}$ Y. Lou, ${ }^{8}$ S. Lucas,${ }^{8}$ F. Martin ${ }^{13}$ B. Montanini, ${ }^{13}$ C. Napoli, ${ }^{26}$ D.R. Nelson, ${ }^{36}$ C. Nelson, ${ }^{37}$ K. Nieminen, ${ }^{31}$ O. Nilsson, ${ }^{12}$ G. Peter, ${ }^{22}$ R.

18 Philippe ${ }^{5}$ G. Pilate,${ }^{23}$ A. Poliakov ${ }^{25}$ J. Razumovskaya, ${ }^{2}$ P. Richardson, ${ }^{8} \mathrm{C}$. Rinaldi, ${ }^{13} \mathrm{~K}$. Ritland, ${ }^{7}$ P. Rouzé, ${ }^{10}$ D. Ryaboy, ${ }^{25}$ J. Schmutz, ${ }^{28}$ J. Schrader ${ }^{38}$ B. Segerman, ${ }^{9}$ H. Shin, ${ }^{11}$ A. Siddiqui, ${ }^{11}$ F. Sterky, ${ }^{39}$ A. Terry, ${ }^{8}$ C. Tsai ${ }^{15}$ E. Uberbacher, ${ }^{2}$ P. Unneberg, ${ }^{39}$ J.

21 Vahala, ${ }^{32}$ K. Wall, ${ }^{18}$ S. Wessler, ${ }^{21}$ G. Yang ${ }^{21}$ T. Yin, ${ }^{1}$ C. Douglas, ${ }^{6 \dagger}$ M. Marra,${ }^{11 \dagger}$ G. Sandberg, ${ }^{12 \dagger}$ Y. Van de Peer, ${ }^{10 \dagger}$ D. Rokhsar, ${ }^{8,24 \dagger}$

${ }^{1}$ Environmental Sciences Division, Oak Ridge National Laboratory, Oak Ridge, TN 37831, USA.

${ }^{2}$ Life Sciences Division, Oak Ridge National Laboratory, Oak Ridge, TN 37831, USA.

$30{ }^{3}$ Plant Sciences Department, University of Tennessee, TN 37996, USA.

${ }^{4}$ Department of Biology, West Virginia University, Morgantown, WV 26506, USA.

${ }^{5}$ Michael Smith Laboratories, University of British Columbia, Vancouver, BC V6T 1Z4, Canada.

${ }^{6}$ Department of Botany, University of British Columbia, Vancouver, BC V6T 1Z4, Canada.

${ }^{7}$ Department of Forest Sciences, University of British Columbia, Vancouver, BC V6T 1Z4, Canada.

${ }^{8}$ U.S. Department of Energy, Joint Genome Institute, Walnut Creek, CA 94598, USA.

45 'Umeå Plant Science Centre, Department of Plant Physiology, Umeå University, SE-901 87, Umeå, Sweden.

$48{ }^{10}$ Department of Plant Systems Biology, Flanders Interuniversity Institute for Biotechnology (VIB), Ghent University, B-9052 Gent, Belgium. 
${ }^{11}$ Genome Sciences Centre, 100-570 West 7th Avenue, Vancouver, BC V5Z 4S6, Canada.

${ }^{12}$ Umeå Plant Science Centre, Department of Forest Genetics and Plant Physiology, Swedish University of Agricultural Sciences, SE-901 83 Umeå, Sweden.

${ }^{13}$ Tree-Microbe Interactions Unit, INRA-Université Henri Poincaré, INRA-Nancy, 54280 Champenoux, France.

${ }^{14}$ Department of Forestry, Virginia Polytechnic Institute and State University, Blacksburg, VA 24061, USA.

${ }^{15}$ Biotechnology Research Center, School of Forest Resources and Environmental Science, Michigan Technological University, Houghton, MI 49931, USA.

16 Department of Cell \& Systems Biology, University of Toronto, 25 Willcocks St., Toronto, Ontario, M5S 3B2 Canada.

${ }^{17}$ School of Forest Resources and Huck Institutes of the Life Sciences, the Pennsylvania State University, University Park, PA 16802, USA.

${ }^{18}$ Department of Biology, Institute of Molecular Evolutionary Genetics, and Huck Institutes of Life Sciences, The Pennsylvania State University, University Park, PA 24 16802, USA.

${ }^{19}$ Architecture et Fonction des Macromolécules Biologiques, UMR6098, CNRS and

27 Universities of Aix-Marseille I \& II, case 932, 163 avenue de Luminy, 13288 Marseille, France.

$30{ }^{20}$ Warnell School of Forest Resources, University of Georgia, Athens, GA 30602, USA.

${ }^{21}$ Department of Plant Biology, University of Georgia, Athens, GA 30602, USA.

${ }^{22}$ School of Forest Resources and Conservation, Genetics Institute, and Plant Molecular and Cellular Biology Program, University of Florida, Gainesville, FL 32611, USA.

${ }^{23}$ Institut National de la Recherche Agronomique -Orléans, Unit of Forest Improvement, Genetics and Physiology, 45166 Olivet Cedex, France.

${ }^{24}$ Center for Integrative Genomics, University of California, Berkeley, CA 94720 , USA.

$42{ }^{25}$ Genomics Division, Lawrence Berkeley National Laboratory, Berkeley, CA 94720, USA.

$45{ }^{26}$ Department of Plant Sciences, University of Arizona, Tucson, AZ 85721, USA.

${ }^{27}$ Department of Biological Sciences, Purdue University, West Lafayette, IN 47907, USA.

${ }^{28}$ The Stanford Human Genome Center and the Department of Genetics, Stanford University School of Medicine, Palo Alto, CA 94305, USA. 
$3{ }^{29}$ Institute of Forest Genetics, United States Department of Agriculture, Forest Service, Davis, CA 95616, USA.

$6 \quad{ }^{30}$ Federal Research Centre for Forests, Hauptstrasse 7, A-1140 Vienna, Austria.

${ }^{31}$ Plant Molecular Biology Laboratory, Institute of Biotechnology, University of Helsinki, 9 Fl-00014 Helsinki, Finland.

${ }^{32}$ Department of Biological and Environmental Sciences, University of Helsinki, FI-00014 12 Helsinki, Finland.

${ }^{33}$ Department of Biology, 200014, University of Turku, Fl-20014 Turku, Finland.

15

${ }^{34}$ Southern Institute of Forest Genetics, United States Department of Agriculture, Forest Service and Department of Forest Science, Texas A\&M University, College Station, TX 1877843 , USA.

${ }^{35}$ Whitehead Institute for Biomedical Research and Department of Biology, 21 Massachusetts Institute of Technology, Cambridge, MA 02142, USA.

${ }^{36}$ Department of Molecular Sciences and Center of Excellence in Genomics and 24 Bioinformatics, University of Tennessee, Memphis, TN 38163 , USA.

${ }^{37}$ Southern Institute of Forest Genetics, United States Department of Argiculture, Forest

27 Service, Saucier, MS 39574, USA.

${ }^{38}$ Developmental Genetics, University of Tübingen, D-72076 Tübingen, Germany.

${ }^{39}$ Department of Biotechnology, KTH, AlbaNova University Center, SE-106 91 Stockholm, Sweden.

33

*These authors contributed equally to this work as second authors.

†These authors contributed equally to this work as senior authors. 


\section{ABSTRACT}

We report the draft genome of the black cottonwood tree, Populus trichocarpa. Integration of shotgun sequence assembly with genetic mapping enabled chromosomescale reconstruction of the genome. Over 45,000 putative protein-coding genes were

6 identified. Analysis of the assembled genome revealed a whole-genome duplication event, with approximately 8,000 pairs of duplicated genes from that event surviving in the Populus genome. A second, older duplication event is indistinguishably coincident

9 with the divergence of the Populus and Arabidopsis lineages. Nucleotide substitution, tandem gene duplication and gross chromosomal rearrangement appear to proceed substantially slower in Populus relative to Arabidopsis. Populus has more protein-coding

12 genes than Arabidopsis, ranging on average between 1.4-1.6 putative Populus homologs for each Arabidopsis gene. However, the relative frequency of protein domains in the two genomes is similar. Overrepresented exceptions in Populus include

15 genes associated with disease resistance, meristem development, metabolite transport and lignocellulosic wall biosynthesis.

18 KEYWORDS: Whole-genome shotgun sequencing, genome-wide duplication, perennial habit, woody plant, poplar, Salix, Arabidopsis, angiosperm evolution 
Forests cover thirty percent of the earth's terrestrial surface (ca., 3.8 billion hectares), harbor large amounts of biodiversity, and provide humanity with benefits,

3 including clean air and water, lumber, fiber and fuels. Worldwide, one quarter of all industrial feedstocks have their origins in forest-based resources(1). Occurring in extensive wild populations across continents, large and long-lived forest trees have

6 evolved under selective pressures unlike those of annual herbaceous plants. Their growth and development involves extensive secondary growth, coordinated signaling and distribution of water and nutrients over great distances, and strategic storage and

9 re-distribution of metabolites in concordance with inter-annual climatic cycles. The need to survive and thrive in fixed locations over centuries under continually changing physical and biotic stresses also sets them apart from short-lived plants. Many of the features

12 that distinguish trees from other organisms, especially their large sizes and longgeneration times, present challenges to the study of the cellular and molecular mechanisms that underlie their unique biology. To enable and facilitate such

15 investigations in a relatively well-studied model tree, we describe here the draft genome of black cottonwood, Populus trichocarpa (Torr. \& Gray) and its comparison with other sequenced plant genomes.

18 Populus trichocarpa was selected as the model forest species for genome sequencing not only because of its modest genome size, but also because of its rapid growth, relative ease of experimental manipulation, and range of available genetic

21 tools $(2,3)$. The genus is phenotypically diverse and interspecific hybrids facilitate the genetic mapping of economically important traits related to growth rate, stature, wood properties and paper quality. Dozens of quantitative trait loci (QTL) are already

24 mapped(4) and methods of genetic transformation have been developed(5). Under appropriate conditions, Populus can reach reproductive maturity in as few as 4-6 years, permitting selective breeding for large-scale sustainable plantation forestry. Finally, rapid

27 growth of trees coupled with thermochemical or biochemical conversion of the lignocellulosic portion of the plant has the potential to provide a renewable energy resource with a concomitant reduction of greenhouse gases(6-8).

\section{SEQUENCING and ASSEMBLY}

A single female genotype, 'Nisqually-1', was selected and used in a whole-

33 genome shotgun sequence and assembly strategy(9). Approximately 7.6 million endreads representing 4.2 billion high-quality (i.e., Q20 or higher) base pairs were 
assembled into 2,447 major scaffolds containing an estimated $410 \mathrm{Mb}$ of genomic DNA (SOM T1 \& T2). On the basis of the depth of coverage of major scaffolds ( 7.5X), and

3 the total amount of non-organellar shotgun sequence that was generated, the Populus genome size was estimated to be $485 \pm 10 \mathrm{Mb}$, in rough agreement with previous cytogenetic estimates of approximately $550 \mathrm{Mb}(10)$. The near completeness of the

6 shotgun assembly in protein-coding regions is supported by the identification of more than $95 \%$ of known Populus cDNA in the assembly (see Gene Content section below).

The $\sim 75 \mathrm{Mb}$ of unassembled genomic sequence is consistent with cytogenetic 9 evidence for $\sim 30 \%$ of the genomic being heterochromatic(9). The amount of euchromatin contained within the Populus genome was estimated in parallel by subtraction on the basis of direct measurements of DAPI-stained prophase and

12 metaphase chromosomes (SOM F4). On average, $69.5 \pm 0.3 \%$ of the genome consisted of euchromatin, with a significantly lower proportion of euchromatin in linkage group I $(66.4 \pm 1.1 \%)$, compared to the other 18 chromosomes $(69.7 \pm 0.03 \%, p \leq 0.05)$. In contrast,

15 Arabidopsis chromosomes contain roughly 93\% euchromatin(11). The unassembled shotgun sequences were derived from variants of organellar DNA, including recent nuclear translocations, highly repetitive genomic DNA, haplotypic segments that were 18 redundant with short subsegments of the major scaffolds (separated due to extensive sequence polymorphism, i.e., allelic variants), and contaminants of the template DNA, including endophytic microbes inhabiting the leaf and root tissues used for template

21 preparation(12) (SOM F1 \& T3). The end reads corresponding to chloroplast (SOM F5) and mitochondrial genomes were assembled into circular genomes of 157 and $803 \mathrm{~kb}$, respectively(9).

24 We anchored the $410 \mathrm{Mb}$ of assembled scaffolds to a sequence-tagged genetic map (SOM F3). In total, 356 microsatellite markers were used to assign 155 scaffolds (335 $\mathrm{Mb}$ of sequence) to the $19 \mathrm{P}$. trichocarpa chromosome-scale linkage groups (LG)

27 (13). The vast majority (91\%) of the mapped microsatellite markers were colinear with the sequence assembly. At the extremes, the smallest chromosome, LGIX (79 cM), is covered by two scaffolds containing $12.5 \mathrm{Mb}$ of assembled sequence; whereas the 30 largest chromosome, LGI (265 cM), contains 21 scaffolds representing $35.5 \mathrm{Mb}$ (SOM F3). We also generated a physical map based on BAC fingerprint contigs using a Nisqually-1 BAC library representing an estimated 9.5-fold genome coverage (SOM F2).

33 Paired BAC-end sequences from most of the physical map were linked to the large-scale assembly, permitting 2,460 of the physical map contigs to be positioned on the genome 
assembly. Combining the genetic and physical map, nearly $385 \mathrm{Mb}$ of the $410 \mathrm{Mb}$ of assembled sequence is placed on a linkage group.

Unlike Arabidopsis, where predominantly self-fertilizing ecotypes maintain low levels of allelic polymorphism, Populus species are predominantly dioecious, which results in obligate outcrossing. This compulsory outcrossing, along with wind pollination

6 and wind dispersed plumose seeds, results in high levels of gene flow and high levels of heterozygosity (i.e., within-individual genetic polymorphisms). Within the heterozygous Nisqually-1 genome, we identified 1,241,251 single nucleotide polymorphisms (SNP) or

9 small indel polymorphisms for an overall rate of approximately 2.6 polymorphisms per $\mathrm{kb}$. Of these polymorphisms the overwhelming majority (83\%) occurr in non-coding portions of the genome (Table 1). Short insertion/deletion polymorphisms (indels) and

12 SNP polymorphisms within exons resulted in some frameshifts and nonsense stop codons within predicted exons, respectively, suggesting that null alleles of these genes exist in one of the haplotypes. Some of the polymorphisms may be artifacts from the

15 assembly process; though these errors were minimized by using stringent criteria for SNP identification(9).

\section{GENE ANNOTATION}

We tentatively identified a first-draft reference set of 45,555 protein-coding gene loci in the Populus nuclear genome (www.jgi.doe.gov/poplar) using a variety of ab initio,

21 homology-based and EST-based methods(14-17) (SOM T5). Similarly, 101 and 52 genes were annotated in the chloroplast and mitochondrial genomes, respectively ${ }^{9}$. To aid the annotation process, 4,664 full-length sequences, from full-length enriched cDNA

24 libraries from Nisqually-1, were generated and used in training the gene-calling algorithms. Prior to gene prediction, repetitive sequences were characterized (SOM F15 \& T14) and masked; additional putative transposable elements were identified and

27 subsequently removed from the reference gene set(9). Given the current draft nature of the genome, we expect that the gene set in Populus will continue to be refined.

Approximately $89 \%$ of the predicted gene models had homology $\left(E\right.$-value $\left.\leq 1 e^{-8}\right)$

30 to the non-redundant (NR) set of proteins from $\mathrm{NCBI}$, including $60 \%$ with extensive homology over $75 \%$ of both model and NR protein lengths. Nearly $12 \%(5,248)$ of the predicted Populus genes had no detectable similarity to Arabidopsis genes (E-value $\leq 1 e^{-}$

$333^{3}$ ); conversely, in the more refined Arabidopsis set, only $9 \%(2,321)$ of the predicted genes had no similarity to the Populus reference set. Of the 5,248 Populus genes 
without Arabidopsis similarity, 1,883 have expression evidence from the manuallycurated Populus EST dataset, and of these, 274 have no hits $\left(E-v a l u e \geq 1 e^{-3}\right)$ to the NR

3 database(9). Whole-genome oligonucleotide microarray analysis provided evidence of tissue-based expression for $53 \%$ for the reference gene models (Fig. 1). In addition, signal was detected from $20 \%$ of genes that were initially annotated and excluded from

6 the reference set, suggesting that as many as 4,000 additional genes (or gene fragments) may be present. Within the reference gene set, 13,019 pairs of orthologs were identified between genes in Populus and Arabidopsis using the best bi-directional

9 BLAST hits, with average mutual coverage of these alignments equal to $93 \% ; 11,654$ pairs of orthologs had coverage greater than $90 \%$ of gene lengths, with only 156 genes with less than $50 \%$ coverage. As of June 1, 2006, 10\% $(4,378)$ gene models have been

12 manually validated and curated.

\section{GENOME ORGANIZATION}

\section{Genome Duplication in the Salicaceae}

Populus and Arabidopsis lineages diverged ca. 100-120 Mya. Analysis of the Populus genome provided evidence of a more recent duplication event that impacted 18 roughly $92 \%$ of the Populus genome. Nearly 8,000 pairs of paralogous genes of similar age (excluding tandem or local duplications) were identified (Fig. 2). The relative age of the duplicate genes was estimated by the accumulated nucleotide divergence at four-

21 fold synonymous third-codon transversion position (4DTV) values. A sharp peak in 4DTV values, corrected for multiple substitutions, representing a burst of gene duplication, is evident at $0.0916 \pm 0.0004$ (Fig. 3A). Comparison of 1,825 Populus and Salix orthologous

24 genes derived from Salix EST suggests that both genera share this whole-genome duplication event (Fig. 3B). Moreover, the parallel karyotypes and collinear genetic maps(18) of Salix and Populus also support the conclusion that both lineages share the

27 same large-scale genome history.

If we naively calibrate the molecular clock using synonymous rates observed in the Brassicaceae(19) or derived from the Arabidopsis-Oryza divergence(20), we would

30 conclude that the genome duplication in Populus is very recent (8-13 Mya as reported by $\left.{ }^{19}\right)$. Yet the fossil record shows that the Populus and Salix lineages diverged 60-65 million years ago(22-25). Thus the molecular clock in Populus must be ticking at only

33 one sixth the estimated rate for Arabidopsis (i.e., 8-13 Mya/60-65 Mya). Qualitatively similar slowing of the molecular clock is found in the Populus chloroplast and 
mitochondrial genomes(9). As a long-lived vegetatively propagated species Populus has the potential to successfully contribute gametes to multiple generations. A single

3 Populus genotype can persist as a clone on the landscape for millennia(26), and we propose that recurrent contributions of "ancient gametes" from very old individuals could account for the dramatically reduced rate of sequence evolution. As result of the slowing

6 of the molecular clock, the Populus genome most likely resembles the ancestral eurosid genome.

To test if the burst of gene creation 60-65 Mya was due to a single whole9 genome event or independent but near-synchronous gene duplication events we used a variant of the algorithm of Hokamp et al. (27) to identify segments of conserved synteny within the Populus genome. The longest conserved syntenic block from the 4DTV $\sim 0.09$

12 epoch spanned 765 pairs of paralogous genes. In total, 32,577 genes were contained within syntenic blocks from the salicoid epoch; half of these genes were contained in segments longer than 142 paralogous pairs. The same algorithm, when applied to

15 randomly shuffled genes, typically yields duplicate blocks with fewer than 8-9 genes, indicating that the Populus gene duplications occurred as a single genome-wide event. Through the remainder of this paper this duplication event will be referred to as the

18 "salicoid" duplication event.

Nearly every mapped segment of the Populus genome had a parallel "paralogous" segment elsewhere in the genome as a result of the salicoid event (Fig. 2).

21 The "pinwheel" patterns can be understood as a whole-genome duplication followed by a series of reciprocal tandem terminal fusions between two separate sets of four chromosomes each; the first involving LGII, V, VII and XIV and the second involving LGI,

$24 \mathrm{XI}$, IV and IX. In addition, several chromosomes appear to have experienced minor reorganizational exchanges. Furthermore, LGI appears to be the result of multiple rearrangements involving three major tandem fusions. These results suggest that the

27 progenitor of Populus had a base chromosome number of 10 which, following the wholegenome duplication event, experienced a genome-wide reorganization and diploidization of the duplicated chromosomes into four pairs of complete paralogous chromosomes

30 (LGVI, VIII, X, XII, XIII, XV, XVI, XVIII \& XIX), two sets of four chromosomes each containing a terminal translocation (LGI, II, IV, V, VII, IX \& XI) and one chromosome containing three terminally joined chromosomes (LGIII with I or XVII with VII). The

33 colinearity of genetic maps among multiple Populus species suggests that the genome reorganization must have occurred prior to the evolution of the modern taxa of Populus. 


\section{Genome Duplication in a Common Ancestor of Populus and Arabidopsis}

3 The distribution of 4DTV values for paralogous pairs of genes also shows that a large fraction of the Populus genome falls in a set of duplicated segments anchored by gene pairs with 4DTV at $0.364 \pm 0.001$, representing the residue of a more ancient, large-scale,

6 apparently synchronous duplication event (Fig 3A). This relatively older duplication event covers approximately $59 \%$ of the Populus genome with $16 \%$ of genes in these segments present in two copies. Since this duplication preceded and is therefore superimposed

9 upon the salicoid event, each genomic region is potentially covered by four such segments. Similarly, the Arabidopsis genome experienced an older "beta" duplication that preceded the Brassicaceae-specific "alpha" event(28-32).

12 We next asked if the Arabidopsis "beta" $(\mathbf{3 0}, 32)$ and Populus 4DTV 0.36 duplication events were (i) independent genome-wide duplications that occurred after the split from the last common eurosid ancestor $\left(\mathrm{H}_{1}\right)$ or (ii) a single shared duplication

15 event that occurred in an ancestral lineage (i.e., prior to the eurosid I/II divergence) $\left(\mathrm{H}_{2}\right)$. These two hypotheses have very different implications for the interpretation of homology between Populus and Arabidopsis. Under $\mathrm{H}_{1}$ each genomic segment in one species is

18 homologous to four segments in the other, while under $\mathrm{H}_{2}$ each segment is homologous to only two segments in the other species. These hypotheses were tested by comparing the relative distances between gene pairs sampled within and between Populus and

21 Arabidopsis. $\mathrm{H}_{2}$ was generally supported(9), but, we could not reject $\mathrm{H}_{1}$. We can only conclude that the Populus genome duplication occurred very close to the time of divergence of the Eurosid I and II lineages(9), with slight support for a shared

24 duplication. This coincident timing raises the possibility of a causal link between this duplication and rapid diversification early in eurosid (and perhaps core eudicot) history. Through the remainder of this paper this older Populus/Arabidopsis duplication event will

27 be referred to as the "eurosid" duplication event. We note that the salicoid duplication occurred independently of the eurosid duplication observed in the Arabidopsis genome.

\section{GENE CONTENT}

Although Populus has substantially more protein-coding genes than Arabidopsis, the relative frequency of domains represented in protein databases (Prints, Prosite,

33 Pfam, ProDom \& SMART) in the two genomes is similar(9). However, the most common domains occur in Populus in a 1.4-1.8 to one ratio compared with Arabidopsis. 
Noteworthy outliers in Populus include genes and gene domains associated with disease and insect resistance (e.g., leucine rich repeats, 1,271 vs. 527; NB-ARC

3 domain, 302 vs. 141; thaumatin, 55 vs. 24, Populus vs. Arabidopsis, respectively), meristem development (e.g., NAC transcription factors, 157 vs. 100, respectively) and metabolite/nutrient transport (e.g., oligopeptide transporter of the POT and OPT families,

6129 vs. 61 ; potassium transporter, 30 vs. 13, respectively).

Some domains were underrepresented in Populus compared to Arabidopsis. For example, the F-box domain was twice as prevalent in Arabidopsis as in Populus (624 vs.

9303 , respectively). The F-box domain is involved in diverse and complex interactions involving protein degradation via the ubiquitin-26S proteosome pathway(33). Many of the ubiquitin-associated domains are underrepresented in Populus compared to Arabidopsis

12 (e.g., Ulp1 protease family, C-terminal catalytic domain, 10 vs. 63, respectively). Moreover, the RING finger domains are nearly equally present in both genomes (503 vs. 407 , respectively), suggesting that protein degradation pathways in the two organisms

15 are metabolically divergent.

\section{The Common Eurosid Gene Set}

18 The Populus and Arabidopsis gene sets were compared to infer the conserved gene complement of their common eurosid ancestor, integrating information from nucleotide divergence, synteny and mutual best BLAST-hit analysis(9). The ancestral

21 eurosid genome contained at least 11,666 protein-coding genes, along with an undetermined number that were either lost in one or both of the lineages or whose homology could not be detected. These ancestral genes were the progenitors of gene

24 families of typically 1-4 descendents in each of the complete plant genomes and account for 28,257 Populus and 17,521 Arabidopsis genes. Gene family lists are accessible at: www.phytozome.net. The gene predictions in these two genomes that could not be

27 accounted for in the eurosid clusters were often fragmentary or could not be confidently assigned orthology and may include novel or rapidly evolving genes in the Populus and/or Arabidopsis lineages, as well as poorly predicted genes.

\section{Non-Coding RNAs}

Based on a series of publicly available RNA detection algorithms(34), including 33 tRNAScan-SE, INFERNAL and snoScan, we identified 817 putative transfer RNAs (tRNA), 22 U1, 26 U2, 6 U4, 23 U5, 11 U6 spliceosomal RNAs (snRNA), 339 putative 
C/D small nucleolar RNAs (snoRNA) and 88 predicted H/ACA snoRNAs in the Populus assembly. All 57 possible anti-codon tRNA were found. One selenocysteine tRNA was detected and two possible suppressor tRNA (anticodons which bind stop codons) were also discovered. Populus has nearly 1.3 times as many tRNA genes as Arabidopsis. In contrast to Arabidopsis (SOM F7A), the copy number of tRNA in Populus was

6 significantly and positively correlated with amino acid occurrence in predicted gene models (SOM F7B). Populus has a 1.3 to 1.0 ratio in the number of snRNA compared with Arabidopsis, yet $\mathrm{U} 1, \mathrm{U} 2$ and $\mathrm{U} 5$ are overrepresented in Populus while $\mathrm{U} 4$ is

9 underrepresented. Furthermore, U14 was not detected in Arabidopsis. The snRNA and snoRNA have not been experimentally verified in Populus.

There are 169 identified microRNA (miRNA) genes representing 21 families in

12 Populus (SOM T7). In Arabidopsis, these 21 families contain 91 miRNA genes, representing a 1.9X expansion in Populus, primarily in miR169 and miR159/319. All 21 miRNA families have regulatory targets that appear to be conserved among Arabidopsis

15 and Populus (SOM T8). Like the miRNA genes themselves, the number of predicted targets for these miRNA is expanded in Populus (147) compared to Arabidopsis (89). Similarly, the genes that mediate RNAi are also overrepresented in Populus (21)

18 compared to Arabidopsis (11) (e.g., AGO1 class, 7 vs. 3; RNA helicase 2 vs.1; HEN, 2 vs. 1 ; HYL1-like (dsRNA binding proteins) 9 vs. 5, respectively).

\section{Tandem Duplications}

In Populus there were 1,518 tandemly duplicated arrays of two or more genes based on a Smith-Waterman alignment E-value $\leq \mathrm{e}^{-25}$ and a $100 \mathrm{~kb}$ window. The total

24 number of genes in such arrays was 4,839 and the total length of tandemly duplicated segments in Populus was $47.9 \mathrm{Mb}$ or $15.6 \%$ of the genome (SOM F8). By the same criteria, there are 1,366 tandemly duplicated segments in Arabidopsis, covering $32.4 \mathrm{Mb}$

27 or $27 \%$ of the genome. By far the most common number of genes within a single array was two, with 958 such arrays in Populus and 805 in Arabidopsis. Arabidopsis had a larger number of arrays containing six or more genes than did Populus. Tandem

30 duplications thus appear to be relatively more common in Arabidopsis than in Populus. This may in part be due to difficulties in assembling tandem repeats from a wholegenome shotgun sequencing approach, particularly when tandemly-duplicated genes

33 are highly conserved. Alternatively, the Populus genome may be undergoing rearrangements at a slower rate than the Arabidopsis genome, which is consistent with 
our observations of reduced chromosomal rearrangements and slower nucleotide substitution rates in Populus.

In some cases, genes were highly duplicated in both species, with some tandem duplications predating the Populus-Arabidopsis split(9). The largest number of tandem repeats in Populus in a single array was 24 and contained genes with high homology to

6 S-locus specific glycoproteins. Genes of this class also occur as tandem repeats in Arabidopsis, with the largest segments containing 14 tandem duplicates on chromosome 1. One of the InterPro domains in this protein, IPR008271, a serine/threonine protein

9 kinase active site, was the most frequent domain in tandemly repeated genes in both species (SOM F8). Other common domains in both species were the leucine-rich repeat (IPR007090, primarily from tandem repeats of disease resistance genes), the

12 pentatricopeptide repeat RNA-binding proteins (IPR002885), and the UDPglucuronosyl/UDP-glucosyltransferase domain (IPR002213) (SOM T9).

In contrast, some genes were highly expanded in tandem duplicates in one

15 genome and not in the other (SOM F8). For example, one of the most frequent classes of tandemly duplicated genes in Arabidopsis was F-box genes, with a total of 342 involved in tandem duplications, of which the largest segment contained 24 F-box

18 genes.. Populus contains only 37 F-box genes in tandem duplications, with the largest segment containing only three genes.

\section{POST-DUPLICATION GENE FATE}

\section{Functional expression divergence}

In Populus, 20 of the 66 salicoid-event duplicate gene pairs contained in 19

24 Populus EST libraries (2.3\% of the total) showed differential expression(9) (displayed significant deviation in EST frequencies per library, e.g., Fig. 4). Eleven of 18 eurosidevent duplicate gene pairs $(2.7 \%$ of the total) also displayed significant deviation in EST

27 frequencies per library. Many of the duplicate gene pairs that displayed significant overrepresentation in one or more of the 19 sampled libraries were involved in proteinprotein interactions (e.g., annexin) or protein folding (e.g., cyclophilins). In the eurosid

30 set, there was a greater divergence in the best BLAST hit among pairwise sets of genes. These results support functional expression divergence among some duplicated gene pairs in Populus.

33 As a further test for variation in gene expression among duplicated genes we examined whole-genome oligonucleotide microarray data containing the 45,555 
promoted genes(9). There was significantly lower differential expression in the salicoid duplicated pairs of genes (mean: 5\%) relative to eurosid duplications (mean: 11\%),

3 again suggesting that differential expression patterns for retained paralogous gene pairs is an ongoing process that has had more time to occur in eurosid pairs (Fig. 5). This difference could also be due to absolute expression level, which may vary systematically

6 between the two duplication events. Moreover, differential expression was more evident in the wood-forming organs. Almost $14 \%$ and $13 \%$ (2,632 pairs of genes) of eurosid duplicated genes in the nodes and internodes, respectively, displayed differential 9 expression compared to $8 \%$ or lower in roots and young leaves (Fig. 5).

\section{Single Nucleotide Polymorphisms}

12 Populus is a highly polymorphic taxon and substantial numbers of SNP are present even within a single individual (Table 1). The ratio of nonsynonymous to synonymous substitution rate $(\omega=\mathrm{dN} / \mathrm{dS})$ was calculated as an index of selective

15 constraints for alleles of individual genes(9). The overall average $\mathrm{dN}$ across all genes was 0.0014 , while dS value was 0.0035 , for a total $\omega$ of 0.40 , suggesting that the majority of coding regions in the Populus genome are subject to purifying selection.

18 There was a significant, negative correlation between $\omega$ and the 4DTV distance to the most closely related paralog $(r=-0.034, p=0.028)$, which is consistent with the expectation of higher levels of nonsynonymous polymorphism in recently duplicated

21 genes due to functional redundancy $(20,35)$. Similarly, genes with recent tandem duplicates $(4 \mathrm{DTV} \leq 0.2)$ had significantly higher $\omega$ than genes with no recent tandem duplicates (Wilcoxon Rank Sum Z=8.65, p $\leq 0.0001$ ) (SOM T10).

24 The results for tandemly duplicated genes were consistent with expectations for accelerated evolution of duplicated genes(20). However, this expectation was not upheld for paralogous pairs of genes from the whole-genome duplication events. Relative rates

27 of nonsynonymous substitution were actually lower for genes with paralogs from the salicoid and eurosid whole-genome duplication events than for genes with no paralogs (SOM T11). One possible explanation for this discrepancy is that the apparent single-

30 copy genes have a corresponding overrepresentation of rapidly-evolving pseudogenes. However, this does not appear to be the case, as demonstrated by an analysis of gene size, synonymous substitution rate and minimum genetic distance to the closest paralog

33 as covariates in an analysis of variance with $\omega$ as the response variable (SOM T11). Therefore, genes with no paralogs from the salicoid and eurosid duplication events seem 
to be under lower selective constraints and purifying selection is apparently stronger for genes with paralogs retained from the whole-genome duplications. Chapman et al. (36)

3 have recently proposed the concept of functional buffering to account for similar reduction in detected mutations in paralogs from whole-genome duplications in Arabidopsis and Oryza. The vegetative propagation habit of Populus may also favor the

6 conservation of nucleotide sequences among duplicated genes, in that complementation among duplicate pairs of genes would minimize loss of gene function associated with the accumulation of deleterious somatic mutations.

9

\section{Gene Family Evolution}

Lignocellulosic Wall Formation Among the processes unique to tree biology, one of

12 the most obvious is the yearly development of secondary xylem from the vascular cambium. Populus orthologs of the approximately 20 Arabidopsis genes/gene families involved in or associated with cellulose biosynthesis were identified. The Populus

15 genome has 93 cellulose synthesis-related genes vs. 78 in Arabidopsis. Arabidopsis genome encodes 10 CesA genes belonging to six classes known to participate in cellulose microfibril biosynthesis(37). Populus has 18 CesA genes(38), including

18 duplicate copies of CesA7 and CesA8 homologs. Populus homologs of Arabidopsis CesA4, CesA7 and CesA8 are coexpressed during xylem development and tension wood formation(39). Furthermore, one pair of CesA genes appears unique to Populus,

21 with no homologs found in Arabidopsis(40). Many other types of genes associated with cellulose biosynthesis, e.g., KOR, SUSY, COBRA and FRA2, occur in duplicate pairs in Populus relative to single-copy Arabidopsis genes(39). For example COBRA, a regulator

24 of cellulose biogenesis(41), is a single-copy gene in Arabidopsis yet in Populus there are four copies.

The repertoire of acknowledged hemicellulose biosynthetic genes in Populus is

27 generally similar to that in Arabidopsis. However, Populus has more genes encoding aL-fucosidases and fewer genes encoding $\alpha$-L-fucosyltransferases than does Arabidopsis, which is consistent with the lower xyloglucan fucose content(42) in Populus

30 relative to Arabidopsis.

Lignin, the second most abundant secondary cell wall polymer after cellulose, is a complex polymer of monolignols (hydroxycinnamyl alcohols) that encrusts and 33 interacts with the cellulose/hemicellulose matrix of the secondary cell wall(43). The full set of 34 Populus phenylpropanoid and lignin biosynthetic genes (SOM T13) were 
identified by sequence alignment to the known Arabidopsis phenylpropanoid and lignin genes $(44,45)$. The size of Populus gene families encoding these enzymes is generally

3 larger than in Arabidopsis (34 vs. 18, respectively). The only exception is CAD (cinnamyl alcohol dehydrogenase), which is encoded by a single gene in Populus and two genes in Arabidopsis (Fig. 6C); CAD is also encoded by only a single gene in Pinus taeda(46,

6 47). Two lignin-related Populus $\mathrm{C} 4 \mathrm{H}$ genes are strongly co-expressed in tissues related to wood formation while the three Populus $\mathrm{C} 3 \mathrm{H}$ genes show reciprocally exclusive expression patterns(48).

Secondary Metabolism Populus produce a broad array of non-structural, carbon-rich secondary metabolites that exhibit wide variation in abundance, stress inducibility, and

12 effects on tree growth and host-pest interactions(49-53). Shikimate-phenylpropanoid derived phenolic esters, phenolic glycosides and condensed tannins and their flavonoid precursors comprise the largest classes of these metabolites. Phenolic glycosides and

15 condensed tannins alone can constitute up to $35 \%$ leaf dry weight and are abundant in buds, bark and roots of Populus $(\mathbf{5 0}, \mathbf{5 4}, \mathbf{5 5})$.

The flavonoid biosynthetic genes are well annotated in Arabidopsis(56) and

18 almost all (with the exception of flavonol synthase) are encoded by single-copy genes. In contrast, all but three such enzymes (chalcone isomerase, flavonoid 3'-hydroxylase and flavanone 3-hydroxylase) are encoded by multiple genes in Populus(53). For example,

21 the chalcone synthase (CHS), controlling the committed step to flavonoid biosynthesis, has expanded to at least six genes in Populus. In addition, Populus contains two genes each for flavone synthase II (CYP98B) and flavonoid 3', 5'-hydroxylase (CYP75A12 and

24 CYP75A13) which are absent in Arabidopsis. Furthermore, three Populus genes encode leucoanthocyanidin reductase, required for the synthesis of condensed tannin precursor 2,3-trans-flavan-3-ols, a stereochemical configuration also lacking in Arabidopsis(57). In

27 contrast to the 32 terpenoid synthases (TPS) genes of secondary metabolism identified in the Arabidopsis genome(58), the Populus genome contains at least 47 TPS genes, suggesting a wide-ranging capacity for the formation of terpenoid secondary 30 metabolites.

A number of phenylpropanoid-like enzymes have been annotated in the Arabidopsis genome (44, 45, 59-61). One example is the family encoding cinnamyl 33 alcohol dehydrogenase (CAD). In addition to the single Populus $C A D$ gene involved in lignin biosynthesis, several other clades of CAD-like (CADL) genes are present, most of 
which fall within larger sub-families containing enzymes related to multifunctional alcohol dehydrogenases (Fig. 6). This comparative analysis makes it clear that there has been

3 selective expansion and retention of Populus CADL gene families. For example, Populus contains seven CADL genes (PoptrCADL1-7; Fig. 6C) encoding enzymes related to the Arabidopsis $B A D 1$ and $B A D 2$ enzymes with apparent benzyl alcohol dehydrogenase

6 activities(62). BAD1 and BAD2 are known to be pathogen-inducible, suggesting that this group of Populus genes, including Populus $S A D$ gene, previously characterized as encoding a sinapaldehyde-specific CAD enzyme(63), may be involved in chemical 9 defense.

Disease Resistance The likelihood that a perennial plant will encounter a pathogen or

12 herbivore before reproduction is near unity. The long-generation intervals for trees make it difficult for such plants to match the evolutionary rates of a microbial or insect pest. Aside from the formation of thickened cell walls and the synthesis of secondary 15 metabolites that constitute a first line of defense against microbial and insect pests, plants use a variety of disease-resistance $(R)$ genes.

The largest class of characterized $R$ genes encodes intracellular proteins that 18 contain a nucleotide-binding site (NBS) and carboxy-terminal leucine-rich-repeats (LRR) (64). The NBS-coding $R$ gene family is one of the largest in Populus, with 399 members, approximately 2 -fold higher than in Arabidopsis. The NBS family can be divided into

21 multiple subfamilies with distinct domain organizations, including 64 TIR-NBS-LRR genes, 10 truncated TIR-NBS that lack an LRR, 233 non-TIR-NBS-LRR genes and 17 unusual TIR-NBS-containing genes not identified previously in Arabidopsis (TNLT, TNLN

24 or TCNL) (Table 2). Five gene models coding for TNL proteins contained a predicted Nterminal nuclear localization signal (NLS) (65). The number of non-TIR-NBS-LRR genes in Populus is also much higher than that in Arabidopsis (209 vs. 57, respectively).

27 Intriguingly, 40 non-TIR-NBS genes, not found in Arabidopsis, carry an N-terminal BED DNA-binding zinc finger domain that was also found in the Oryza Xa1 gene. These findings suggest that domain cooption occurred in Populus. Most NBS-LRR (ca. 65\%) in

30 Populus occur as singletons or in tandem duplications and the distribution of pairwise genetic distances among these genes suggests a recent expansion of this family. That is, only $10 \%$ of the NBS-LRR genes are associated with the eurosid and salicoid 33 duplication events, compared with $55 \%$ of the extracellular LRR receptor-like kinase genes, for example (SOM F10). 
Several conserved signaling components such as RAR1, EDS1, PAD4 and NPR1, known to be recruited by $R$ genes, also contain multiple homologs in Populus.

3 For example, two copies of the PAD4 gene, which functions upstream of salicylic acid (SA) accumulation, and five copies of the NPR1, an important regulator of responses downstream of SA, are found in Populus. Nearly all genes known to control disease

6 resistance signaling in Arabidopsis have putative orthologs in Populus. Populus has a larger number of $\beta-1,3-$ glucanase and chitinase genes than Arabidopsis (131 vs. 73, respectively). In summary, the structural and genetic diversity that exists among $R$ genes

9 and their signaling components in Populus is remarkable and suggests that unlike the rest of the genome, contemporary diversifying selection has played an important role in the evolution of disease resistance genes in Populus. Such diversification suggests that

12 enhanced ability to detect and respond to biotic challenges via $R$ gene-mediated signaling may be critical over a decades-long life span of this genus.

Membrane Transporters Attributes of Populus biology such as massive interannual,

15 seasonal and diurnal metabolic shifts and re-deployment of carbon and nitrogen may require an elaborate array of transporters. Investigation of gene families coding for transporter proteins (http://plantst.genomics.purdue.edu/) in the Populus genome

18 revealed a general expansion relative to Arabidopsis (1,722 vs. 959, Populus vs. Arabidopsis, respectively) (SOM T12). Five gene families, coding for ATP-binding cassette proteins (ABC transporters, 226 gene models), major facilitator superfamily

21 proteins (MFS, 187 genes), drug/metabolite transporters (DMT, 108 genes), amino acid/auxin permeases (AAAP, 95 genes) and proton-dependent oligopeptide transporters (POT, 90 genes), accounted for more than $40 \%$ of the total number of

24 transporter gene models (SOM F14). Some large families such as those encoding POT (4.3X relative to Arabidopsis), glutamate-gated ion channels (3.7X), potassium uptake permeases (2.3X) and $A B C$ transporters (1.9X) are expanded in Populus. A novel

27 subfamily of five putative aquaporins, lacking in the Arabidopsis, was identified. Populus also harbors seven transmembrane receptor genes only found so far in fungi, and two genes, identified as mycorrhizal-specific phosphate transporters, confirming that the

30 mycorrhizal symbiosis may have a significant impact on the mineral nutrition of this longlived species. This expanded inventory of transporters could conceivably play a role in adaptation to nutrient-limited forest soils, long-distance transport and storage of water 
and metabolites, secretion and movement of secondary metabolites, and/or mediation of resistance to pathogen-produced secondary metabolites or other toxic compounds.

Phytohormones Both physiological and molecular studies have indicated the importance of hormonal regulation underlying plant development. Auxin, gibberellin,

6 cytokinin and ethylene responses are of particular interest in tree biology.

Many auxin responses(66-71) are controlled by auxin response factor (ARF) transcription factors, which work together with cognate AUX/IAA repressor proteins to

9 regulate auxin-responsive target genes(72, 73). A phylogenetic analysis using the known and predicted ARF protein sequences showed that Populus and Arabidopsis ARF gene families have expanded independently since they diverged from their common ancestor.

12 Six duplicate ARF genes in Populus encode paralogs of ARF genes that are single-copy Arabidopsis genes, including ARF5 (MONOPTEROS), an important gene required for auxin-mediated signal transduction and xylem development. Furthermore, five

15 Arabidopsis ARF genes have four or more predicted Populus ARF gene paralogs. In contrast to ARF genes, Populus does not contain a dramatically expanded repertoire of AUXIIAA genes relative to Arabidopsis (35 vs. 29, respectively) (74). Interestingly, there

18 is a group of four Arabidopsis AUX/IAA genes with no apparent Populus orthologs, suggesting Arabidopsis-specific functions.

Gibberellins are thought to regulate multiple processes during wood and root

21 development, including xylem fiber length(75). Among all gibberellin biosynthesis and signaling genes, the Populus GA20-oxidase gene family is the only family with approximately 2 -fold increase in gene number relative to Arabidopsis, indicating that

24 most of the duplicated genes that arose from the salicoid duplication event have been lost. GA20-oxidase appears to control flux in the biosynthetic pathway leading to the bioactive gibberellins $\mathrm{GA}_{1}$ and $\mathrm{GA}_{4}$. The higher complement of GA20-oxidase genes

27 may have biological significance in Populus with respect to secondary xylem and fiber cell development.

Cytokinins are thought to control the identity and proliferation of cell types

30 relevant for wood formation as well as general cell division(67). The total number of members in gene families encoding cytokinin homeostasis related isopentenyl transferases (IPT) and cytokinin oxidases is roughly similar between Populus and

33 Arabidopsis, although there appears to be lineage-specific expansion of IPT subfamilies. The cytokinin signal transduction pathway represents a two-component phosphorelay 
system, where a two-component hybrid receptor initiates a phosphotransfer via histidinecontaining phosphotransmitters (HPt) to phospho-accepting response regulators $(R R)$.

3 One family of genes, encoding the two-component receptors (i.e., CKI1), is notably expanded in Populus (4 vs. 1, respectively) (76). Gene families coding for recently identified pseudo HPt and atypical RR are overrepresented in Populus relative to

6 Arabidopsis (2.5X and 4.0X, respectively). Both of these gene families have been implicated in the negative regulation of cytokinin signaling $(67,77)$, which is consistent with the idea of increased complexity in regulation of cytokinin signal transduction in 9 Populus.

Populus and Arabidopsis genomes contain almost identical number of genes for the three enzymes of ethylene biosynthesis, whereas the number of genes for proteins

12 involved in ethylene perception and signaling is higher in Populus. For example, Populus has seven predicted genes for ethylene receptor proteins and Arabidopsis has five; the constitutive triple response (CTR1) kinase that acts just downstream of the receptor is

15 encoded by four genes in Populus and only one in Arabidopsis(78). The number of ethylene-responsive element binding factor (ERF) proteins (a subfamily of $A P 2 / E R F$ family) is higher in Populus than in Arabidopsis (172 vs. 122, respectively). The

18 increased variation in the number of ERF transcription factors may be involved in the ethylene-dependent processes specific to trees, such as tension wood formation(68) and the establishment of dormancy(71).

\section{CONCLUDING REMARKS}

Our initial analyses provide a flavor of the opportunities for comparative plant 24 genomics made possible by the generation of the Populus genome sequence. A complex history of whole-genome duplications, chromosomal rearrangements and tandem duplications has shaped the genome that we observe today. The differences in

27 gene content between Populus and Arabidopsis have provided some tantalizing insights into the possible molecular bases of their strongly contrasting life histories, though it is important to note that factors unrelated to gene content (e.g., regulatory elements,

30 miRNA, post-translational modification, or epigenetic modifications) may ultimately be of equal or greater importance. With the sequence of Populus, researchers can now go beyond what could be learned from Arabidopsis alone to explore hypotheses to linking

33 genome sequence features to wood development, nutrient and water movement, crown development, and disease resistance in perennial plants. The availability of the Populus 
genome sequence will enable continuing comparative genomics studies among species that will shed new light on genome reorganization and gene family evolution.

3 Furthermore, the genetics and population biology of Populus make it an immense source of allelic variation. Because Populus is an obligate outcrossing species, recessive alleles tend to be maintained in a heterozygous state. Informatics tools enabled by the

6 sequence, assembly and annotation of the Populus genome will facilitate the characterization of allelic variation in wild Populus populations adapted to a wide range of environmental conditions and gradients over large portions of the northern

9 hemisphere. Such variants represent a rich reservoir of molecular resources useful in biotechnological applications, development of alternative energy sources, and mitigation of anthropogenic environmental problems. Finally, the keystone role of Populus in many

12 ecosystems provides the first opportunity for the application of genomics approaches to questions with ecosystem-scale implications $(\mathbf{7 9}, \mathbf{8 0})$. 


\section{Support References and Notes}

1. FAO. State of the World's Forests 2003. 2003. Rome, Food and Agricultural Organization of the United Nations. State of the World's Forests (SOFO) - SOFO 2003.

Ref Type: Serial (Book,Monograph)

2. R. F. Stettler, Jr. H. D. Bradshaw, in Biology of Populus and its implications for management and conservation, R. F. Stettler, Jr. H. D. Bradshaw, P. E. Heilman, T. M. Hinckley, Eds. (NRC Research Press, Ottawa, 1996) ,chap. Overview, pp. $1-6$.

3. G. A. Tuskan, S. P. DiFazio, T. Teichmann, Plant Biology 6, 2 (2004).

12 4. T. M. Yin, S. P. DiFazio, L. E. Gunter, D. Riemenschneider, G. A. Tuskan, Theoretical and Applied Genetics 109, 451 (2004).

5. R. Meilan, D. Ellis, G. Pilate, A. M. Brunner, J. Skinner, in Forest Biotechnology: Scientific Opportunities and Social Challenges., S. H. Strauss, Jr. H. D. Bradshaw, Eds. (Resources for the Future Press, Washington, D.C., 2004), pp. 36-51.

18 6. G. A. Tuskan, Biomass \& Bioenergy 14, 307 (1998).

7. G. A. Tuskan, Forestry Chronicle 77, 259 (2001).

8. S. Wullschleger et al., Canadian Journal of Forest Research-Revue Canadienne de Recherche Forestiere 35, 1779 (2005).

9. See Supplemental Materials for further information.

10. H. D. Bradshaw, R. F. Stettler, Theoretical and Applied Genetics 86, 301 (1993).

24 11. M. Koornneef, P. Fransz, H. de Jong, Chromosome Research 11, 183 (2003).

12. O. Santamaria, J. J. Diez, Forest Pathology 35, 95 (2005).

13. G. A. Tuskan et al., Canadian Journal of Forest Research-Revue Canadienne de Recherche Forestiere 34, 85 (2004).

14. A. A. Salamov, V. V. Solovyev, Genome Research 10, 516 (2000).

15. E. Birney, R. Durbin, Genome Research 10, 547 (2000).

30 16. T. Schiex, A. Moisan, P. Rouze, in Computational Biology: selected papers from JOBIM'2000 number 2066 in LNCS, Springer-Verlag, Ed. 2001), pp. 118-133.

17. Y. Xu, E. C. Uberbacher, Journal of Computational Biology 4, 325 (1997).

33 18. S. J. Hanley, M. D. Mallot, A. Karp, Tree Genetics and Genomics, in press. 
19. M. A. Koch, B. Haubold, T. Mitchell-Olds, Molecular Biology and Evolution 17, $1483(2000)$.

3 20. M. Lynch, J. S. Conery, Science 290, 1151 (2000).

21. L. Sterck et al., New Phytologist 167, 165 (2005).

22. L. A. Dode, Bulletin de la Societe d'Historie Naturelle d'Autun 18, 161 (1905).

6 23. R. Regnier, Revue des Sociétés Savantes de Normandie (1956).

24. M. E. Collinson, Proceedings of the Royal Society of Edinburgh Section BBiological Sciences 98, 155 (1992).

$925 . \quad$ J. E. Eckenwalder, in Biology of Populus and its implications for management and conservation, R. F. Stettler, Jr. H. D. 1. Bradshaw, P. E. Heilman, T. M. Hinckley, Eds. (NRC Research Press, Ottawa, 1996) ,chap. 1, pp. 7-32.

12 26. J. B. Mitton, M. C. Grant, Bioscience 46, 25 (1996).

27. K. Hokamp, A. McLysaght, K. H. Wolfe, J. Struct. Funct. Genomics 3, 95 (2003).

28. J. E. Bowers, B. A. Chapman, J. K. Rong, A. H. Paterson, Nature 422, 433

15 (2003).

29. L. M. Zahn, H. Kong, J. H. Leebens-Mack, S. Kim, P.S. Soltis, L. L. Landherr, D.E. Soltis, C. W. dePamphilis, H. Ma, Genetics 169, 2209-2223 (2005).

18 30. S. De Bodt, S. Maere, Y. Van de Peer, Trends in Ecology \& Evolution 20, 591 (2005).

31. K. L. Adams, J. F. Wendel, Trends in Genetics 21, 539 (2005).

21 32. G. Blanc, K. Hokamp, K. H. Wolfe, Genome Research 13, 137 (2003).

33. B. A. Schulman et al., Nature 408, 381 (2000).

34. S. Griffiths-Jones et al., Nucleic Acids Research 33, D121 (2005).

24 35. S. Lockton, B. S. Gaut, Trends in Genetics 21, 60 (2005).

36. B. A. Chapman, J. E. Bowers, F. A. Feltus, A. H. Paterson, PNAS 103, 2730 (2006).

27 37. T. A. Richmond, C. R. Somerville, Plant Physiology 124, 495 (2000).

38. S. Djerbi, M. Lindskog, L. Arvestad, F. Sterky, T. T. Teeri, Planta 221, 739 (2005).

30 39. C. P. Joshi et al., New Phytologist 164, 53 (2004).

40. A. Samuga, C. P. Joshi, Gene 334, 73 (2004). 
41. F. Roudier et al., Plant Cell 17, 1749 (2005).

42. R. M. Perrin et al., Science 284, 1976 (1999).

3 43. R. W. Whetten, J. J. Mackay, R. R. Sederoff, Annual Review of Plant Physiology and Plant Molecular Biology 49, 585 (1998).

44. J. Ehlting et al., Plant Journal 42, 618 (2005).

45. J. Raes, A. Rohde, J. H. Christensen, P. Y. Van de, W. Boerjan, Plant Physiol 133, 1051 (2003).

46. D. M. O'Malley, S. Porter, R. R. Sederoff, Plant Physiology 98, 1364 (1992).

9 47. J. J. Mackay, W. W. Liu, R. Whetten, R. R. Sederoff, D. M. O’Malley, Molecular \& General Genetics 247, 537 (1995).

48. J. Schrader et al., Plant Cell 16, 2278 (2004).

12 49. S. Whitham, S. McCormick, B. Baker, Proceedings of the National Academy of Sciences of the United States of America 93, 8776 (1996).

50. G. M. Gebre, T. J. Tschaplinski, G. A. Tuskan, D. E. Todd, Tree Physiology 18, 645 (1998).

51. G. Arimura, D. P. W. Huber, J. Bohlmann, Plant Journal 37, 603 (2004).

52. D. J. Peters, C. P. Constabel, Plant Journal 32, 701 (2002).

18 53. C.-J. Tsai, S. A. Harding, T. J. Tschaplinski, R. L. Lindroth, Y. Yuan, New Phytologist 172: 47-62 (2006).

54. M. M. De Sá, R. Subramaniam, F. E. Williams, C. J. Douglas, Plant Physiology $2198,728(1992)$.

55. R. L. Lindroth, S. Y. Hwang, Biochemical Systematics and Ecology 24, 357 (1996).

24 56. B. Winkel-Shirley, Curr. Opin. Plant Biol. 5, 218 (2002).

57. G. J. Tanner et al., Journal of Biological Chemistry 278, 31647 (2003).

58. S. Aubourg, A. Lecharny, J. Bohlmann, Molecular Genetics and Genomics 267, 730 (2002).

59. M. A. Costa et al., Phytochemistry 64, 1097 (2003).

60. D. Cukovic, J. Ehlting, J. A. VanZiffle, C. J. Douglas, Biological Chemistry 382, 645 (2001).

61. J. M. Shockey, M. S. Fulda, J. Browse, Plant Physiology 132, 1065 (2003). 
62. I. E. Somssich, P. Wernert, S. Kiedrowski, K. Hahlbrock, Proceedings of the National Academy of Sciences of the United States of America 93, 14199 (1996).

63. L. G. Li et al., Plant Cell 13, 1567 (2001).

64. B. C. Meyers, S. Kaushik, R. S. Nandety, Curr. Opin. Plant Biol. 8, 129 (2005).

65. L. Deslandes et al., Proceedings of the National Academy of Sciences of the United States of America 99, 2404 (2002).

66. E. J. Mellerowicz, M. Baucher, B. Sundberg, W. Boerjan, Plant Molecular Biology 47, 239 (2001).

9 67. A. P. Mähönen et al., Science 311, 94 (2006).

68. S. Andersson-Gunneras et al., Plant Journal 34, 339 (2003).

69. J. M. Hellgren, K. Olofsson, B. Sundberg, Plant Physiology 135, 212 (2004).

12 70. M. G. Cline, K. Dong-II, Annals of Botany 90, 417 (2002).

71. R. Ruonala, P.L.H. Rinne, M. Baghour, T. Moritz, H. Tuominen, J. Kangasjärvi, Plant Journal 46, 628 (2006).

15 72. R. Moyle et al., Plant Journal 31, 675 (2002).

73. D. Weijers et al., Embo Journal 24, 1874 (2005).

74. G. Hagen, T. Guilfoyle, Plant Molecular Biology 49, 373 (2002).

18 75. M. E. Eriksson, M. Israelsson, O. Olsson, T. Moritz, Nature Biotechnology 18, 784 (2000).

76. T. Kakimoto, Science 274, 982 (1996).

21 77. T. Kiba, K. Aoki, H. Sakakibara, T. Mizuno, Plant and Cell Physiology 45, 1063 (2004).

78. T. Nakano, K. Suzuki, T. Fujimura, H. Shinshi, Plant Physiology 140, 411 (2006).

79. Acknowledgments - The authors wish to thank U.S. Department of Energy, Office of Science for supporting the sequencing and assembly portion of this study, Genome Canada and the Province of British Columbia for providing support for the BAC end, BAC genotyping, and full-length CDNA portions of this study, the Swedish Agricultural University for supporting the EST assembly and annotation portion of this study, the membership of the International Populus Genome Consortium for supplying genetic and genomics resources used in the assembly and annotation of the genome, the National Science Foundation, Plant Genome Program for supporting the development of web-based tools, Drs. Toby Bradshaw and Reinhold Stettler for input and reviews on draft copies of the manuscript, Mr. Jason Tuskan for guidance and input during the analysis and 
writing of the manuscript, and to the anonymous reviewers who provided critical input and recommendations on the manuscript.

3 80. GenBank Accession Number: AARH00000000 